\title{
Minor actinide fission induced by multi-nucleon transfer reaction in inverse kinematics
}

\author{
X. Derkx ${ }^{1, a}$, F. Rejmund ${ }^{1}$, M. Caamaño ${ }^{1}$, K.-H. Schmidt ${ }^{2}$, L. Audouin ${ }^{3}$, C.-O. Bacri ${ }^{3}$, G. Barreau ${ }^{4}$, J. Benlliure ${ }^{5}$, E. \\ Casarejos $^{5}$, B. Fernández-Domínguez ${ }^{6}$, L. Gaudefroy ${ }^{7}$, C. Golabek ${ }^{1}$, B. Jurado ${ }^{4}$, A. Lemasson ${ }^{1}$, A. Navin $^{1}$, M. Rejmund ${ }^{1}$, \\ T. Roger ${ }^{1}$, A. Shrivastava ${ }^{1}$, C. Schmitt ${ }^{1}$, and J. Taieb ${ }^{7}$ \\ 1 GANIL, CEA/DSM - CNRS/IN2P3, F-140796 Caen, France \\ 2 Gesellschaft für Schwerionenforshung, Planckstrasse 1, 64291 Darmstadt, Germany \\ 3 Institut de Physique Nucléaire, CNRS/IN2P3, F-91406 Orsay, France \\ 4 CENBG, UMR 5797 CNRS/IN2P3, Université Bordeaux 1, F-33175 Gradignan, France \\ 5 Universidad de Santiago de Compostela, 15706 Santiago de Compostela, Spain \\ 6 Oliver Lodge Laboratory, University of Liverpool, Liverpool L69 7ZE, United Kingdom \\ 7 CEA, DAM, DIF, F-91297 Arpajon, France
}

\begin{abstract}
In the framework of nuclear waste incineration and design of new generation nuclear reactors, experimental data on fission probabilities and on fission fragment yields of minor actinides are crucial to design prototypes. Transfer-induced fission has proven to be an efficient method to study fission probabilities of actinides which cannot be investigated with standard techniques due to their high radioactivity. We report on the preliminary results of an experiment performed at GANIL that investigates fission probabilities with multi-nucleon transfer reactions in inverse kinematics between a ${ }^{238} \mathrm{U}$ beam on a ${ }^{12} \mathrm{C}$ target. Actinides from $\mathrm{U}$ to $\mathrm{Cm}$ were produced with an excitation energy range from 0 to $30 \mathrm{MeV}$. In addition, inverse kinematics allowed to characterize the fission fragments in mass and charge. A key point of the analysis resides in the identification of the actinides produced in the different transfer channels. The new annular telescope SPIDER was used to tag the target-like recoil nucleus of the transfer reaction and to determine the excitation energy of the actinide. The fission probability for each transfer channel is accessible and the preliminary results for ${ }^{238} \mathrm{U}$ are promising.
\end{abstract}

\section{Introduction}

Minor actinides are currently produced in the U-Pu electronuclear fuel cycle. Due to their high radioactivity and their low fissibility, they involve risks and are considered as ultimate wastes. Instead of deep geological repository, two other options are worthwhile investigating : the reduction of their production in new fuels cycles (e.g. based on Th-U) and their incineration by transmutation in sub-critical reactors. In both cases, data such as fission cross sections or isotopic distribution of the fission fragments are required to design dedicated facilities. Fission process represents also a great opportunity to improve the understanding of fundamental aspects in nuclear physics.

The standard studies of these actinides rely on their direct neutron irradiation to measure $(\mathrm{n}, \mathrm{f})$ cross section. However such method is limited to long life-time and low activity isotope targets. An indirect way, the "surrogate method", was developped in the 70's by Cramer and Britt [1]. It consists in the production of the same compound nucleus by an alternative reaction with enough excitation energy to enable it to fission. The surrogate reaction is usually a nucleon transfer reaction between a handleable actinide

\footnotetext{
${ }^{\text {a }}$ e-mail: derkx@ganil.fr
}

nearby the desired one and a light nucleus : e.g. (d,p) or $\left({ }^{3} \mathrm{He}, \alpha\right)$ reactions.

The surrogate method calls in the statistical HauserFeshbach theory, in which the formation and decay of the compound nucleus are independent of each other for a given angular momentum $J$ and parity $\pi$. Hence, in this frame, the fission probabilities do not dependent of the formation mechanism of the compound nucleus but of the population of its $(J, \pi)$ states by this mechanism. The populated $(J, \pi)$ states are a priori not the same in the case where the compound nucleus is formed by transfer reaction and in the one where it is formed by neutron capture.

Besides, in the Weisskopf-Ewing limit of the statistical Hauser-Feshbach theory, the fission probabilities are independent of the angular momentum and parity of the compound nucleus and must be thereby the same for neutron capture and transfer reaction. The validity of this approximation the surrogate method required is subject of debate [2].

To expand the range of investigated nuclei, it is possible to transfer more nucleons by using a heavier nuclei like ${ }^{12} \mathrm{C}$. It was shown that $\left({ }^{12} \mathrm{C},{ }^{8} \mathrm{Be}_{\text {g.s. }}\right)$ reaction provides fission probabilities in good agreement with (n,f) reactions [3]. Hence the validity of the method can be expanded to higher

This is an Open Access article distributed under the terms of the Creative Commons Attribution-Noncommercial License, which permits unrestricted use, distribution, and reproduction in any noncommercial medium, provided the original work is properly cited. 


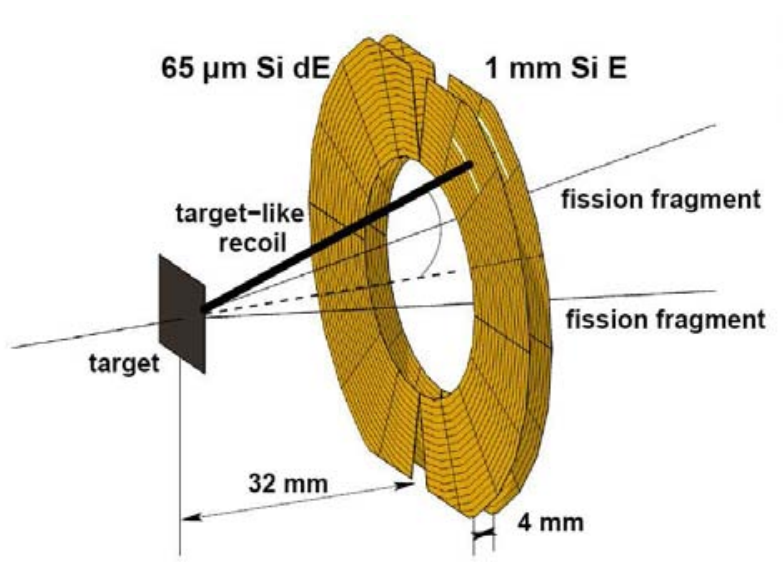

Fig. 1. Scheme of the annular telescope SPIDER

transfer which give access to heavier actinides and allow to study several nuclides in a single experiment.

In the present work, the reaction can be performed in inverse kinematics with an actinide beam and a light target. It avoids parasitic transfer reactions between the incident light nucleus and the impurities present in the actinide target (e.g. Oxygen from actinide oxydes or other isotopes of the nucleus of interest). Moreover, the inverse kinematics leads to a forward focusing of the fission fragments with a high velocity, which improves the detection efficiency and allows a better resolution for their identification.

\section{Experiment}

The experiment was performed at GANIL in April 2008. $\mathrm{A}{ }^{238} \mathrm{U}$ beam impinged a ${ }^{12} \mathrm{C}$ target with an energy of $6.1 \mathrm{~A}$ $\mathrm{MeV}$, i.e. around $10 \%$ above the Coulomb barrier and the target had a thickness of $100 \mu \mathrm{g} . \mathrm{cm}^{-2}$.

A new detector called SPIDER (Silicon Particle Identification DEtector Ring) was used to detect and identify the target-like recoils and to reconstruct the kinematics of the transfer reaction (cf. Fig. 1). It is composed by two annular double-sided silicon detectors Micron S1 [4]. Both detectors have a diameter of $96 \mathrm{~mm}$ and an inner hole of $48 \mathrm{~mm}$. They are also segmented into 16 rings of $1.5 \mathrm{~mm}$ on one side and into 16 sectors on the other one. The first detector is $65 \mu \mathrm{m}$ thick and measures the energy loss $\Delta E$ while the second one is $1 \mathrm{~mm}$ thick and measures the residual energy $E_{\text {res }}$. SPIDER was centred on the beam axis, $32 \mathrm{~mm}$ after the target and a $4 \mathrm{~mm}$ gap separated the $E_{\text {res }}$ detector from the $\Delta E$ one. The angular coverage was between $35^{\circ}$ and $55^{\circ}$, which was at the edge of the grazing angle $\left(\theta_{\text {lab }}^{\text {grazing }}(\right.$ recoil $\left.)=35.7^{\circ}\right)$ where the transfer cross-section is maximal [5].

Several actinides from $\mathrm{U}$ to $\mathrm{Cm}$ were detected with an excitation energy range from 0 to $30 \mathrm{MeV}$ (fonctions of the transfer channel). If the excitation energy was high enough, these actinides may have fissionned. Their in-flight fission fragments were confined in a cone of about $25^{\circ}$ in the laboratory frame due to the inverse kinematics. Hence they were not intercepted by SPIDER and one of them may have

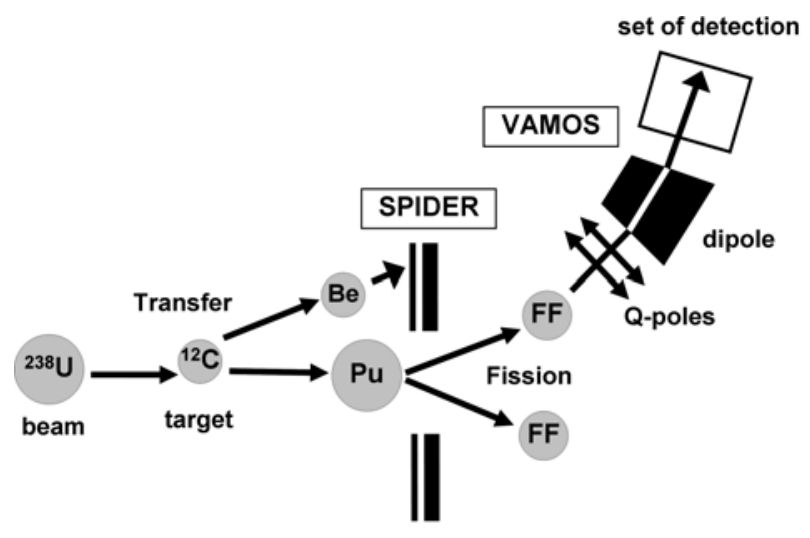

Fig. 2. Experimental set-up

entered the large accepteance spectrometer VAMOS [6] which was rotated by $20^{\circ}$ relatively to the beam axis - to be identified by its set of detectors [7]. A general overview of the experimental set-up is presented in Fig. 2.

The identification and the distribution of the fission fragments are reported in Ref. [8]. At last, two clovers of the Germanium-array EXOGAM [9] were placed around the target to identify nuclear levels in the fission fragments [10].

\section{Preliminary results}

\subsection{Transfer channel identification}

The actinides produced by transfer reactions are tagged by the detection and the identification of the target-like recoil nuclei in SPIDER. $\triangle E-E_{\text {res }}$ correlations are presented in Fig. 3 with the identification of $\mathrm{C}, \mathrm{B}, \mathrm{Be}, \mathrm{Li}$ and $\mathrm{He}$. The corresponding actinides produced are $\mathrm{U}, \mathrm{Np}, \mathrm{Pu}, \mathrm{Am}$ and $\mathrm{Cm}$. Nevertheless there is no isotopic identification.

The lack of isotopic separation is due to several technical issues. The main one concerns the high rate of radiation in SPIDER (up to 40.000 particles. s $^{-1}$ on $\Delta E$ part), mostly from the elastic scattering of the ${ }^{12} \mathrm{C}$ target, which generated high leaking current. As a consequence, effective high voltage supply was reduced and the detector was not fully depleted. So the response of SPIDER varied in time, as a function of the beam intensity and current fluctuations. We corrected this behaviour by following the instant centre of gravity of the elastic scattering target events and then by correcting all the events. However the leaking current also deteriotated drastically the resolution by increasing the noise.

Furthermore the interaction of the beam on the target produced a high flux of $\delta$ electrons, which increased the counting rate and induced false triggers. This made loose correlations between the two sides of the $\Delta E$ part of SPIDER and complicated the reconstruction for the events with a multiplicity higher than one.

In addition, the beam wobbled on the horizontal plane with an amplitude of about $3 \mathrm{~mm}$ and a frequency of $1 \mathrm{~Hz}$. This modified the interaction-point on the target and the value of the associated angle thereby, which introduced a spread of the energy deposit in the $\Delta E$ part. 


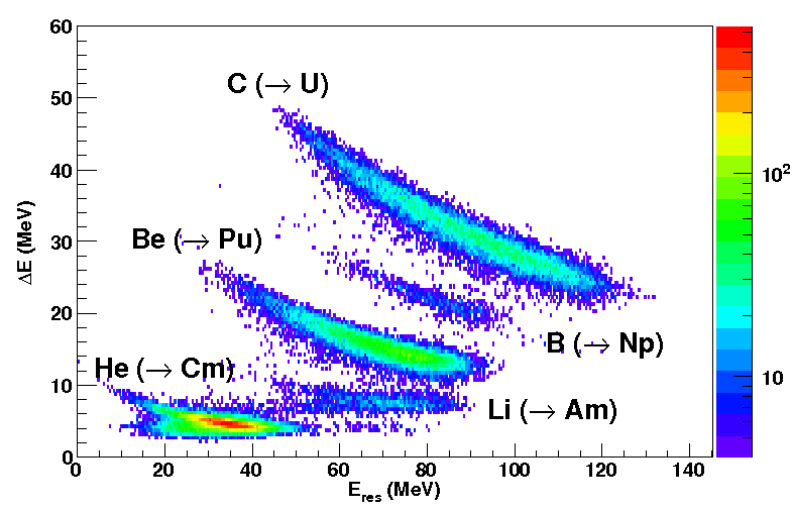

Fig. 3. $\Delta E-E_{\text {res }}$ correlations in SPIDER. The target-like recoils identified are shown and the corresponding produced actinides are indicated in parenthesis.

\subsection{Actinide excitation energy}

The excitation energy of the produced actinides is extracted from a kinematics calculation as a function of the total energy and the angle in the laboratory frame of the target-like recoil nucleus and of the reaction $\mathrm{Q}$ value. The total kinetics energy of the target-like recoil is measured in SPIDER, its angle is determined by the segmentation of the detector. As the system is very asymmetric, the excitation energy is supposed to be principally in the heavy beam-like nucleus which has a higher level of density and much more degree of freedom than the light target-like recoil. So, we assume that the excitation energy of the target-like recoil nucleus is zero.

As there is no isotopic identification, it is impossible to determine the reaction $\mathrm{Q}$ value. However, as the different isotopes are populated in proportion that exponentially depends on the corresponding reaction $Q$ value [11], we may estimate the most probable transfers for each proton channel. These estimations are presented in Tab. 1.

$\mathrm{U}$ and $\mathrm{Np}$ channels are rather clean whereas there is a strong contributions of different isotopes for $\mathrm{Pu}$ and $\mathrm{Am}$ channels. This isotope mixing is an extra source of uncertainties and its impact on the excitation energy resolution is not evaluated yet.

Table 1. Estimation of the most probable transfer channels

\begin{tabular}{lll}
\hline proton/neutron transferred & actinide produced & weight \\
\hline $0 \mathrm{p} / 0 \mathrm{n}$ & ${ }^{238} \mathrm{U}$ & $\lesssim 100 \%$ \\
$0 \mathrm{p} /-1 \mathrm{n}$ & ${ }^{237} \mathrm{U}$ & a few $\%$ \\
\hline $1 \mathrm{p} / 0 \mathrm{n}$ & ${ }^{239} \mathrm{~Np}$ & $\lesssim 100 \%$ \\
$1 \mathrm{p} / 1 \mathrm{n}$ & ${ }^{240} \mathrm{~Np}$ & $\mathrm{a}$ few $\%$ \\
\hline $2 \mathrm{p} / \mathrm{n}$ & ${ }^{240} \mathrm{Pu}$ & $\sim 70 \%$ \\
$2 \mathrm{p} / 1 \mathrm{n}$ & ${ }^{241} \mathrm{Pu}$ & $\sim 30 \%$ \\
\hline $3 \mathrm{p} / 2 \mathrm{n}$ & ${ }^{243} \mathrm{Am}$ & $\sim 60 \%$ \\
$3 \mathrm{p} / 3 \mathrm{n}$ & ${ }^{244} \mathrm{Am}$ & $\sim 40 \%$ \\
\hline
\end{tabular}

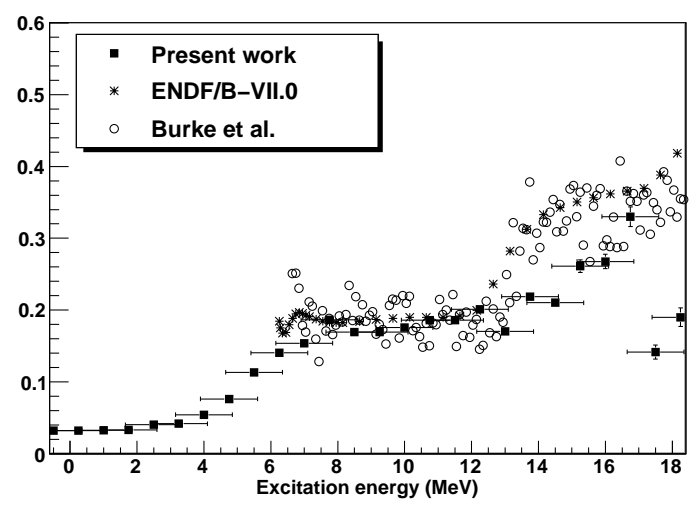

Fig. 4. Preliminary fission probability from ${ }^{12} C\left({ }^{238} U,{ }^{238} U^{\prime}\right){ }^{12} C^{\prime}$ compared with ${ }^{238} \mathrm{U}\left(\alpha, \alpha^{\prime} \mathrm{f}\right)$ [12] and ${ }^{237} \mathrm{U}(\mathrm{n}, \mathrm{f})$ from ENDF/BVII.0

\subsection{Fission probability}

From excitation energy spectra, we can extract the fission probabilties of the produced actinides for each proton channel identified. It consists in making the ratio of the excitation energy spectrum of the chosen actinide gated by the detection of a fission fragment in VAMOS over its excitation energy spectrum in any case.

The excitation energy spectra in coincidence with the detection of a fission fragment have to be corrected of the acceptance of VAMOS which depends on the velocity of the fissionning system. This velocity depends on its excitation energy and on its angle. The determination of this correction is still preliminary. The preliminary result for the fission of ${ }^{238} \mathrm{U}$ induced by inelastic scattering is presented in Fig. 4 with statistical error bars and a preliminary estimated binning in excitation energy.

We compare this result with available experimental data from ${ }^{238} \mathrm{U}\left(\alpha, \alpha^{\prime} \mathrm{f}\right)[12]$ and with evaluated data on ${ }^{237} \mathrm{U}(\mathrm{n}, \mathrm{f})$ from ENDF/B-VII.0. Our data are in quite good agreement with both of these set of data : the relative height of the plateaus of the first and second chance fission match with the data used as reference and the foot of our second plateau is at a compatible energy with these data. Nevertheless the slope from the first to the second plateau is smoother in our case and seems to be as smooth as the slope of our first plateau.

These preliminary results also indicate that the method we use is valuable to study minor actinide fission. The fission probabilities of $\mathrm{Np}, \mathrm{Pu}$ and $\mathrm{Am}$ are still very prelimlinary and are not reported in this proceedings.

\section{Conclusions and perspectives}

The experiment we performed led to the production of actinides from $\mathrm{U}$ to $\mathrm{Cm}$ by multi-nucleon transfer reactions. There is no isotopic identification of the fissionning system but the preliminary results of this very first experiment with such a set-up are encouraging. At least, fission probabilities of most produced actinides are accessible. Our pre- 
liminary results indicate that the multi-nucleon transfer reactions may be an adapted tool for surrogate method for nuclear data.

A new experiment was accepted and will be performed next year at GANIL. A new SPIDER telescope will be used with an upgraded electronics, a cooling system and a protection system against electromagnetic radiations. A test in real condition will be performed before to reach a better resolution for isotopic identification of actinides and their excitation energy.

\section{Acknowledgement}

This work has been supported by Région Basse-Normandie and GEDEPEON, as well as by the EURATOM programme under the contract number 44816.

\section{References}

1. J. D. Cramer and H. C. Britt, Nucl. Sci. Eng. 41, (1970) 177-187

2. J. E. Escher and F. S. Dietrich, Phys. Rev. C 74, (2006) 054601

3. E. Cheifetz et al, Phys. Rev. C 24, (1981) 519-522

4. http://www.micronsemiconductor.co.uk/pdf/s.pdf

5. D. C. Biswas et al, Phys. Rev. C 56, (1997) 1926-1935

6. H. Savajols, Nucl. Phys. A 654, (1999) 1027c-1032c

7. S. Pullanhiotan et al, Nucl. Inst. Meth. A 593, (2008) 343-352

8. M. Caamaño, F. Rejmund et al, to be published in the Proceedings of the 4th International Workshop on $\mathrm{Nu}-$ clear Fission and Fission-Fragment Spectroscopy (2009)

9. F. Azaiez and W. Korten, Nucl. Phys. News 7, issue 4 (1997) 21-25

10. A. Shrivastava et al, submitted to Phys. Rev. C Rapid Comm.

11. J. S. Karp et al, Phys. Rev. C 25, (1982) 1838-1864

12. J. T. Burke et al, Phys. Rev. C 73, (2006) 054604 